\title{
A Mobile Kalman-Filter Based Solution for the Real-Time Estimation of Spatio-Temporal Gait Parameters
}

\author{
Alberto Ferrari, Pieter Ginis, Michael Hardegger, Filippo Casamassima, \\ Laura Rocchi, and Lorenzo Chiari, Member, IEEE
}

\begin{abstract}
Gait impairments are among the most disabling symptoms in several musculoskeletal and neurological conditions, severely limiting personal autonomy. Wearable gait sensors have been attracting attention as diagnostic tool for gait and are emerging as promising tool for tutoring and guiding gait execution. If their popularity is continuously growing, still there is room for improvement, especially towards more accurate solutions for spatio-temporal gait parameters estimation. We present an implementation of a zero-velocity-update gait analysis system based on a Kalman filter and off-the-shelf shoe-worn inertial sensors. The algorithms for gait events and step length estimation were specifically designed to comply with pathological gait patterns. More so, an Android app was deployed to support fully wearable and stand-alone real-time gait analysis. Twelve healthy subjects were enrolled to preliminarily tune the algorithms; afterwards sixteen persons with Parkinson's disease were enrolled for a validation study. Over the 1314 strides collected on patients at three different speeds, the total root mean square difference on step length estimation between this system and a gold standard was $\mathbf{2 . 9 \%}$. This shows that the proposed method allows for an accurate gait analysis and paves the way to a new generation of mobile devices usable anywhere for monitoring and intervention.
\end{abstract}

Index Terms-Gait analysis, inertial sensors, Kalman filter, mobile health, Parkinson's disease, sensor fusion, spatio-temporal gait parameters.

\section{INTRODUCTION}

$\mathbf{G}$ AIT is a semi-automatic motor task that can be highly altered in several musculoskeletal and neurological conditions (e.g., Parkinsonian or hemiplegic gait) [1], [2]. Gait disturbances may be continuous or episodic. Continuous disturbances refer to stable alterations in the walking pattern such as asymmetry, reduced foot clearance, short shuffling steps, high stride-to-stride variability, and stooped trunk posture; episodic

Manuscript received January 07, 2015; revised June 09, 2015; accepted July 11, 2015. Date of publication July 30, 2015; date of current version July 14 2016. The research leading to these results has received funding from the European Union-Seventh Framework Programme (FP7/2007-2013) under the Grant 288516 (CuPiD project)

A. Ferrari, F. Casamassima, L. Rocchi, and L. Chiari are with the Department of Electrical, Electronic and Information Engineering-Guglielmo Marconi, University of Bologna, 40136 Bologna, Italy (e-mail: alberto.ferrari@unibo.it).

P. Ginis is with the Department of Rehabilitation Sciences, Neuromotor Rehabilitation Research Group, KU Leuven, B-3001 Leuven, Belgium.

M. Hardegger is with the Wearable Computing Laboratory, ETH Zürich, 8092 Zürich, Switzerland.

Digital Object Identifier 10.1109/TNSRE.2015.2457511 disturbances occur occasionally and include, among others, festination and start hesitation [3], [4]. Both types of disturbances cause, to different extents, functional disabilities and limitations to mobility with severe consequences on participation in everyday activities and quality of life [5].

There is an increasing body of evidence that supports the use of external stimuli, e.g., rhythmic cues or closed-loop sensory biofeedback, for motor rehabilitation. Recent reviews [1], [6] demonstrate how closed-loop biofeedback on performance during repetitive, high-intensity and task-specific practice, can be effective in restoring motor function. That is indeed the main objective of the $\mathrm{CuPiD}$ project [7], which aims to develop new at-home, rehabilitation-like training modalities to improve gait and mobility for persons with Parkinson's Disease (PD).

The first prerequisite for monitoring locomotor performance and designing tutoring systems that are able to generate timely and appropriate feedbacks about users' gait, is the availability of wearable sensing and computing systems that acquire and real-time process gait signals. One major aim of such systems is the identification of gait cycles, i.e., the estimation of distinctive events such as initial contacts (ICs) and foot offs (FOs) [8], [9]. From the knowledge of ICs and FOs it is possible to obtain temporal gait parameters, e.g., stride duration or cadence [9]. Many approaches to locate ICs and FOs have been proposed in literature, which differ in terms of accuracy and setup complexity. However, as highlighted in a recent review [10], many of them require multiple inertial measurement units (IMUs), sometimes in combination with other sensors such as pressure insoles or foot switches. More so, most of them have been developed for laboratory settings, not allowing for autonomous donning/doffing, real-time detection, and implementation in closedloop architectures [10], [11]. While most articles present a clear picture of which features correspond to ICs and FOs in inertial signals (acceleration, angular velocity), very few of them describe the design of algorithms that are able to perform real-time event identification in online streams of inertial data.

Different is the status quo for spatial gait parameters. The state of the art for accurate and real-time methods allowing the estimation of, e.g., step length and gait speed, is indeed far from being satisfactory [8], [12]. In particular, two main approaches have been proposed: the use of a biomechanical model [13], and strap-down integration [14].

Methods based on models of the lower limbs, such as the double pendulum, require multiple sensors, typically on shanks and thighs, at the expenses of practical usability. Moreover, they 
rely on the assumption of having the subjects' kinematics restricted to the sagittal plane, limiting the accuracy in the presence of irregular gait patterns such as those expressed by persons with motor disabilities [8].

On the other hand, strap-down integration methods determine the IMU orientation from angular rate integration, and subsequently the position from the double integration of acceleration produced by the movement. However, gyroscopes and accelerometers suffer from measurement errors and corresponding integration drifts that severely limit pose estimation during long-term measurements. Typically, by simply doubleintegrating the acceleration after subtracting gravity, the position error grows cubically in time in the short term [15]. Yet, by placing the IMUs on the feet drift can be effectively corrected on a step-by-step basis by exploiting the constraint that feet keep zero velocity $(\mathrm{ZV})$ during the second rocker (i.e., the period of the stance phase in which the shank rolls over the ankle joint [16]) [17]. This correction is known as "zero velocity update" (ZUPT) and was first proposed in the field of gait analysis by Sabatini et al. [14] and then further developed by others [18], [19].

These latter implementations can be ascribed to the broader discipline named as pedestrian dead-reckoning (PDR). PDR is an emerging field of research focusing on highly accurate, robust, and infrastructure-free pedestrian localization in real-time. It is already employed in security and first responders application to achieve mapping, situational awareness and managing of an individual's actions and interactions [20].

The most common method for applying ZUPT to gait data is the Kalman filter (KF) [20]. In linear Gaussian estimation tasks, a KF finds the best state estimate of a dynamic system by minimizing the variance of the estimation error on the base of noisy measurements and a system model (see Section II-E). The KF at each sample computes the state error covariance matrix as covariances and cross-covariances of position, velocities and orientation error terms. Thanks to cross-covariance terms between sensor velocity, position and orientation, the filter is able to effectively correct position and orientation during a $\mathrm{ZV}$ phase [20].

In order to bound position error growth, the accurate detection of the ZUPT interval, that is the ZV instants, is therefore crucial. In fact, as a general rule, the more accurate the ZUPT detection, the more effective are the KF corrections [21]. The ZUPT phase is commonly detected by means of a likelihood ratio test on the base of magnitudes and variances of sensor accelerations and angular velocities along time. As outlined in [22], performances of ZUPT detectors vary significantly with gait speed.

The second prerequisite for monitoring and tutoring users' gait performance is the porting of PDR systems on mobile devices such as smartphones or embedded systems. Implementing step length estimation algorithms on a fully wearable and standalone system, such as a smartphone, has the great advantages of pervasiveness, ubiquity and exploitation of common apps usage experience [6], [23], [24]. The choice of off-the-shelf smartphones and inertial sensors would also keep the system costs low, supporting massive deployment.

To the authors' knowledge, there is no prior literature on the implementation of a real-time PDR on a mobile device able to

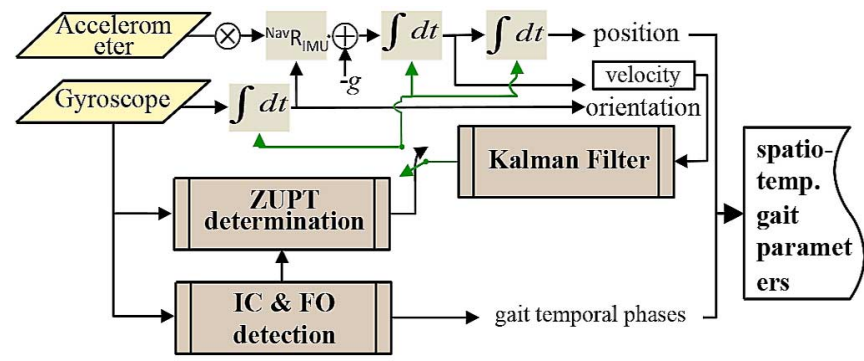

Fig. 1. Flow chart of the PDR system with its three main modules: the algorithms for IC and FO detection and for ZUPT determination, and the KF. ${ }^{\mathrm{Nav}} \mathrm{R}_{\mathrm{IMU}} 3 \times 3$ rotation matrix to transform the accelerations from sensor frame to navigation frame. $\oplus$ indicates a sum; $\otimes$ a cross product and $\int$ integration in time.

accurately detect spatio-temporal gait parameters. In particular, the only method for estimating such parameters which has been validated so far on pathological gait [18] makes the hypothesis to have persons walking on a flat surface and runs offline on a PC. Furthermore, the strap-down integration method used does not exploit the potential of the KF.

In the effort of overcoming current limitations, the aim of this study was two-fold. First, we aimed to design and to validate a PDR system based on shoe-worn IMUs and a KF that can provide accurate spatio-temporal parameters estimation both on normal and pathological gait patterns. Second, we aimed to a fully wearable (i.e., able to run in real-time on a smartphone) PDR system.

To accomplish these goals, a new algorithm able to accurately estimate ICs and FOs has been developed. Based on the gait events detected, the ZUPT algorithm was enhanced in terms of adaptability to abnormal gait patterns and different gait speeds. Finally, the algorithms and the IMU communication software were ported as a library in an Android smartphone, thus realizing a fully wearable PDR system.

\section{METHODS}

This section is organized as follows: first a thorough description of the system architecture and its components is provided from Section II-A to Section II-F. Then in Sections II-G and Section II-H the materials and methods of two distinct experiments (\#1 and \#2) are reported. Experiment \#1 took place during a first phase of the algorithms development process, involved healthy volunteers and served as a benchmark for tuning the PDR parameters with respect to the hardware used in this study. Experiment \#2 was aimed to provide a proof of concept towards the evaluation of system accuracy on a population with gait disturbances.

\section{A. PDR System Architecture}

The overall architecture of the PDR system is presented in Fig. 1. The main modules of the system are illustrated in the following sections: algorithms for IC and FO detection (Section II-C), ZUPT algorithm (Section II-D), and the KF framework (Section II-E). Finally, system implementation on a smartphone is presented (Section II-F). 


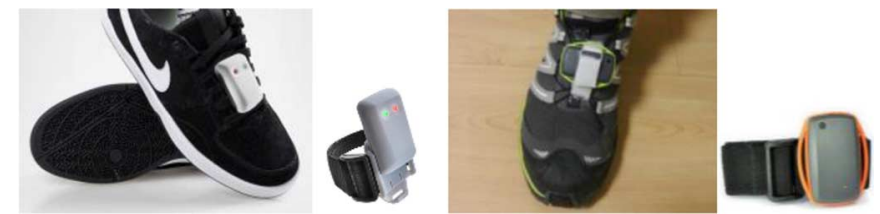

Fig. 2. EXLs3, on the left, and EXLs1 on the right sensing units.

\section{B. Sensors Features and Setup}

In clinical gait analysis with wearable sensors, especially in patients as frail as in PD, it is essential to keep the number of IMUs as low as possible. This ensures usability, preserves adherence and avoids discomfort or stigma. To this aim, after specific focus groups with healthy subjects and patients with PD, we designed a sensing system that makes use of only two IMUs worn on shoes (EXEL, Bologna, IT, Fig. 2). Two subsequent releases of the IMUs (EXLs1, EXLs3) were then used in two different experiments (see Section II-G and Section II-H).

Both releases hold, embedded in the sensor node, a microcontroller, a Bluetooth module, 1 Gb NAND FLASH memory, a Li-ion battery (with a lifetime of $\sim 6 \mathrm{~h}$ ) [25], and differ in terms of the MEMS sensors: the EXLs1 is equipped with the ST LIS3LV02DL and the ST L3G4200D from ST Microelectronics (full scales at $\pm 6 \mathrm{~g}$ and $\pm 2000^{\circ} / \mathrm{s}$ ); the EXLs3 with the MPU9150 from InvenSense (full scales at $\pm 8 \mathrm{~g}$ and $\left.\pm 1000^{\circ} / \mathrm{s}\right)$. In both models the sampling frequency was programmable. The EXLs1 was attached to shoelaces with a customized Velcro strap, whilst a user-friendly holder set with a clipping mechanism allowed for an easy donning/doffing of the EXLs3 sensors. Both EXLs1 and EXLs3 accelerometers were calibrated before usage such that their magnitudes on static acquisitions equaled gravity in the main six directions of space.

\section{Algorithm for IC and FO Detection}

As outlined in [26], very few of the methods proposed in literature to detect IC and FO comply with the scenario's specifications described in Section II-B.

For this reason, we designed a novel algorithm which builds on signals made available from a pair of shoe-worn IMUs and is robust to pathological gait patterns such as those observed in persons suffering from PD. Similar to the algorithm presented by Hundza et al. [8], the algorithm here proposed processes the angular velocity signal arising along the medio-lateral axis of the foot and exploits the feature for which, within each gait cycle, the foot alternatively rotates clockwise and counterclockwise about the ankle joint. In particular, positive peaks (foot rotating counterclockwise when looking at a person walking from her/his right side) are associated with mid-swing events [16]. Then, within each pair of mid-swing peaks, a first (in time) negative peak (foot rotating clockwise) is associated with IC, while a second negative peak is associated with FO (Fig. 3). Unlike from [8], our algorithm runs in real-time and requires shoe-worn IMUs.

Events identification exploits the MATLAB built-in function findpeaks (The Mathworks Inc., Natick, MA, USA) and is based on thresholds and conditions that peaks have to comply with to

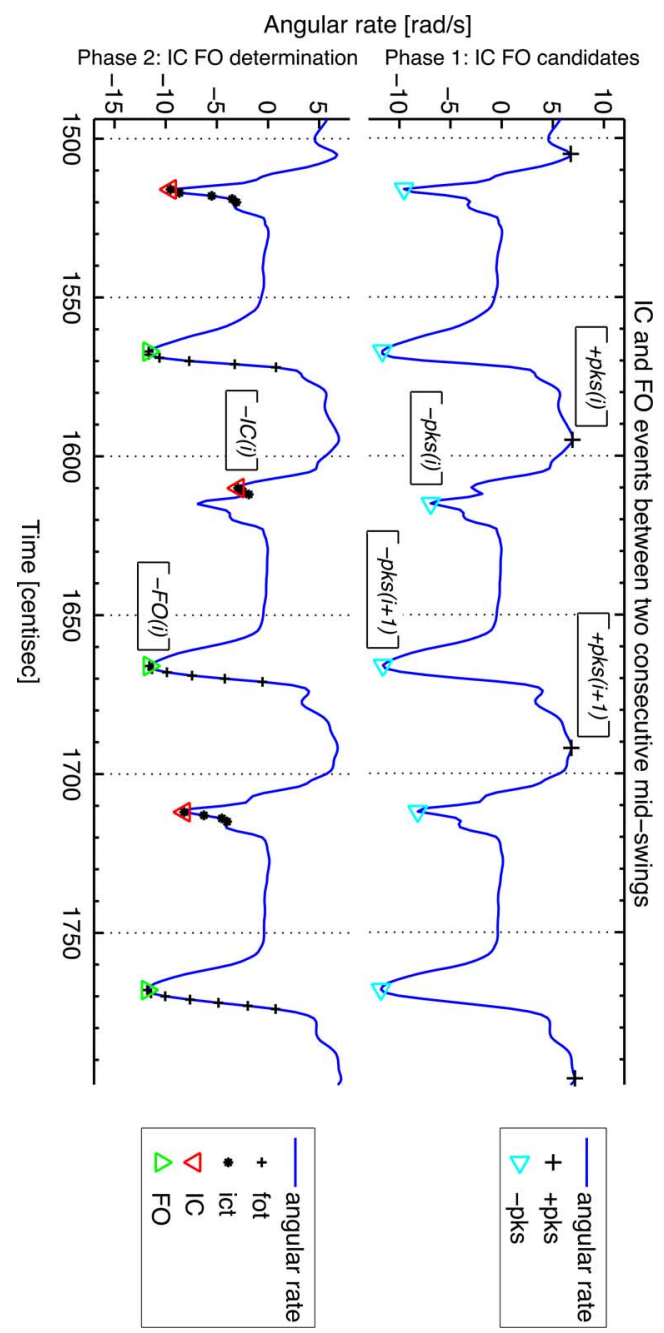

Fig. 3. Phase 1 and 2 of the algorithm for the IC and FO detection (see Section II-C). On the upper subplot, on the signal corresponding to the medio-lateral component of the angular velocity obtained from an EXLs1 during an acquisition of four strides, depiction of the mid-swing events $(\overline{+p k s}$, large plus signs) and the IC and the FO candidates (-pks, right-pointing triangles). On the lower subplot, on the same angular velocity signal, depiction of the $\overline{\imath c t}$ and $\overline{f o t}$ (small plus signs) markers (see Algorithm 1, Section II-C) and of the IC (downward-pointing triangles) and of the FO (upward-pointing triangles).

be recognized as IC or FO. These are summarized in the following pseudo code of Algorithm 1 and sketched in Fig. 3.

Algorithm 1 runs in a soft real-time, with a fixed maximum delay of half a step, which is compliant with mobile gait analysis applications such as feeding back cues during walking in closed loop modality.

From the knowledge of IC and FO instants, it is possible to define the duration of stride, stance and swing phases and consequently all others temporal gait parameters.

\section{ZUPT Algorithm}

ZUPT detectors implement a binary hypothesis testing. They pick out one of the two hypotheses $H 0$ : \{IMU is moving $\}$ and $H 1$ : $\{$ IMU is stationary $\}$. Exploiting the comparative analysis presented in [22], in the present study we implemented the angular rate energy (ARE) detector. ARE measures the energy 


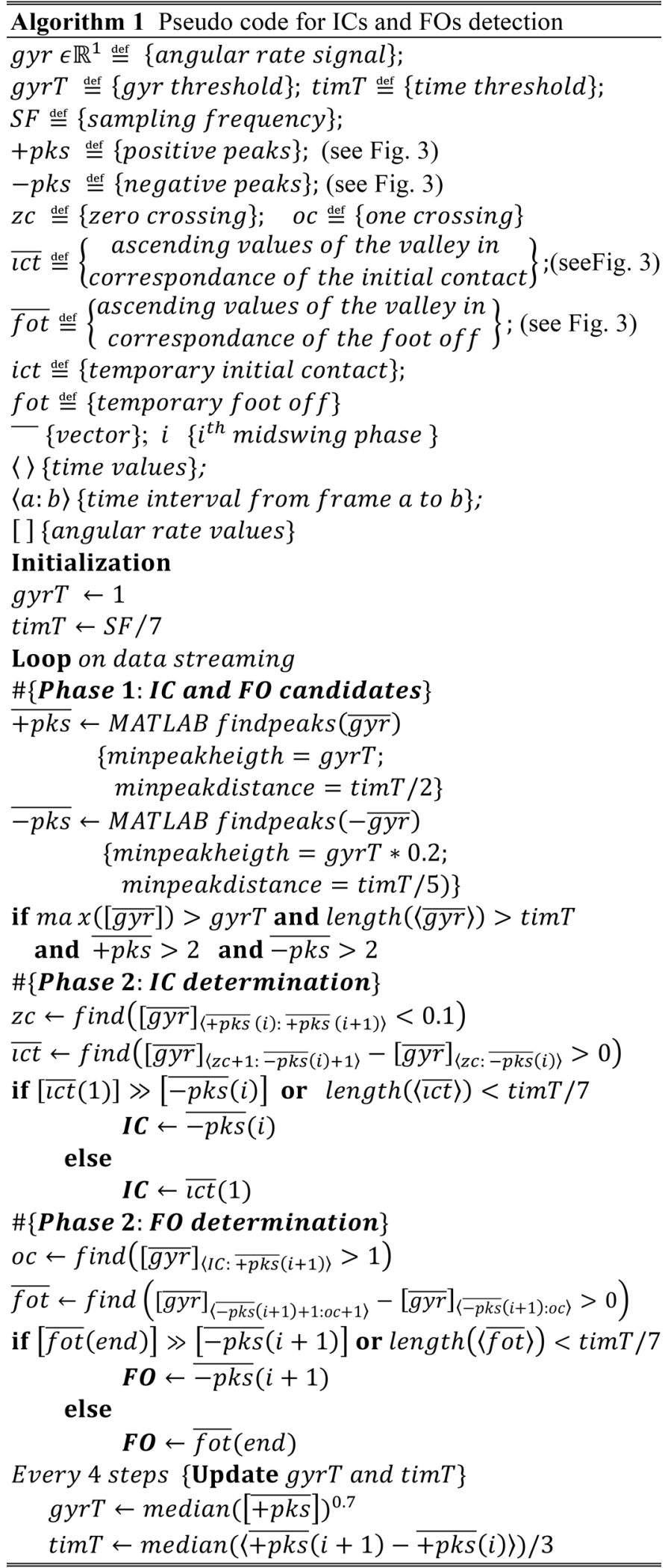

in the gyroscope signal during a time epoch of $\mathrm{W}$ samples and $H 1$ is true whenever the energy is below a threshold $\gamma$ [22]:

$y_{n}^{\omega} \in \mathbb{R}^{3}\{$ angular rate vector at time instant $n\}$ $z_{n}^{\omega} \stackrel{\text { def }}{=}\left\{y_{k}^{\omega}\right\}_{k=n}^{n+W-1}$ \{measurement sequence $\}$

$$
\begin{aligned}
& T\left(z_{n}^{\omega}\right)=\frac{1}{\sigma_{\omega}^{2} W} \sum_{k=n}^{n+W-1}\left\|y_{k}^{\omega}\right\|^{2} \\
& \mathrm{H}_{1}: \mathrm{T}\left(\mathrm{z}_{\mathrm{n}}^{\omega}\right)<\gamma .
\end{aligned}
$$

Usually, the values of $\mathrm{W}, \gamma$ and $\sigma_{\omega}$ are determined empirically by measuring the maximum value of the gyroscope energy in a stationary acquisition. A good estimation of $\mathrm{W}, \gamma$ and $\sigma_{\omega}$ is fundamental for an accurate performance of the ZUPT detector and consequently the KF (see Section II-E).

As shown in [22], gait speed (and pattern) influences ZUPT performances. For example, an increase in gait speed from 5 to 7 $\mathrm{km} / \mathrm{h}$ results in a reduction of final position accuracy by a factor of two. Hence, in order to further improve the accuracy in ZUPT estimation, the ARE algorithm was here enhanced by making its internal parameters automatically adapting to the magnitude of mid-swing events $\overline{+p k s}$ which, in turn, are linearly correlated to gait speed [8]. In particular, every four strides, the current values of W, $\gamma$ and $\sigma_{\omega}$ are updated on the base of $\overline{+p k s}$, following the equations:

$$
\begin{aligned}
\sigma_{\omega} & \leftarrow \frac{\pi}{180 * \mathrm{SF}} * \operatorname{median}([\overline{+p k s}(i-3: i)])^{1.5} \\
W & \leftarrow \frac{\operatorname{median}(\langle\overline{+p k s}(i-3: i)\rangle)}{30} \\
\gamma & \leftarrow 10^{4} * \text { median }([\overline{+p k s}(i-3: i)]) .
\end{aligned}
$$

Finally, in every lapse of time from FO to the following IC, a ZUPT has strictly not to occur. We hence forced the condition

$$
H_{0} \leftarrow\{\langle F O(i): I C(i+1)\rangle\} .
$$

Threshold values and conditional expressions reported in Algorithm 1 and in (2), (3), and (4) are strictly dependent on the IMU hardware and scale settings used in this study and are not straightforwardly applicable with different sensors, yet they can be effectively used as starting point to tune parameters of detectors with different setups.

\section{E. KF Algorithm}

The KF here implemented is based on the work presented in [17]. The KF was first prototyped in MATLAB in form of a GUI allowing for online and offline data acquisition and processing. The offline version was used to tune parameters and data processing procedures; still the same accuracy was maintained in the online version. The simplest implementation of PDR implies five steps: 1) transform accelerations from sensor frame to navigation frame by integrating the angular velocities; 2 ) subtract the gravity; 3 ) integrate accelerations to obtain velocities; 4) reset velocity to zero during ZUPTs; 5) integrate velocity to obtain position [20].

Generally speaking, the KF estimates the state of a system based on noisy measurements and a system model. In this tracking problem, the "system" is the complex of the shoe, the IMU, and the integration equations; the "state" is the error in velocity, position and orientation estimates; and the "measurements" are the zero-velocity phases. Of all possible filters, the $\mathrm{KF}$ is the one that minimizes the variance of the estimation errors, assuming Gaussian-distributed noise. Specifically, in pedestrian dead-reckoning, the KF improves the performances 
of the above described five-step process by exploiting the fact that velocity and position errors are correlated [15]. If during a zero-velocity phase, according to navigation equations, the estimated velocity is not zero, KF does not simply reset the estimated velocity to zero, but also corrects the accumulated error in the estimated position. In other words, KF also estimates the error covariance and cross-covariance terms for position and orientation, hence enabling the filter to correct the position minimizing the variance of the estimation errors.

\section{F. Implementation of the PDR System on a Smartphone}

To increase the pervasiveness of PDR systems it is necessary to make them fully wearable. Exploiting smartphones as mobile processing units and using only two shoe-worn IMUs, enables end-users to handle the system autonomously during daily life.

Gait event detection and PDR algorithms described in Section II-C, Section II-D, and Section II-E have been adapted to run on the dual-core smartphone Samsung S3Mini (OS: Android 4.0 or higher). The software was written in Java and included in Android as a library. The Android application implements the following components.

1. Background services for Bluetooth communication

2. User interface thread.

3. Computational thread (runs the gait analysis algorithms).

4. Data logging thread.

5. Telemedicine service for remote data upload.

MATLAB prototype codes were ported to Java to be compatible with the Android OS. In order to deal with reduced computational performance of the mobile platform, some optimizations were introduced such as the use of floating point instead of double precision arrays for non-critical variables and the allocation of just the memory strictly necessary to maximally reduce its size. The differences between MATLAB- and Java-based software versions were computed on the base of a shared stream of data acquired on a normal subject wearing an EXLs1 during a single walking of 100 steps performed at a comfortable velocity. For each frame acquired during this trial, the differences relative to the walking direction, the medio-lateral and the vertical directions are reported in a probability plot for normal distribution.

\section{G. Experiment \#1: Tuning of PDR Parameters}

A preliminary pilot study was performed on young healthy individuals to i) tune algorithms parameters, ii) verify the agreement between the spatial gait parameters measured by the new system and by a camera-based system, and iii) assess dependency on gait speed.

1) Subjects: Eleven healthy volounteers (7 male), mean (SD) age of 24.3 (6.1) years, were asked to walk over a treadmill (Polar5090, JKexer, Taiwan) at 1.8, 2.7, and $3.5 \mathrm{~km} / \mathrm{h}$ for $1 \mathrm{~min}$. These speeds were set to match the typical ones of patients with PD [26]. An EXLs3 was worn on a shoe of participants. All subjects provided informed consent prior to the start of the study.

2) Data Validation: A retroreflective marker was attached to the IMU and tracked with a videocamera (GoPro HD2 set at a resolution of $1920 \times 1080$ pixels and $30 \mathrm{fps}$ ) assuring similar accuracy to conventional optical motion tracking systems [27]. The ICs were identified and the step lengths estimated from the video footage, similar to [28].

\section{H. Experiment \#2: System Accuracy and Testing Different Sensors Settings}

A second experiment was performed on a sample of subjects with gait disorders, namely people with mild to moderate PD in order to evaluate 1) the robustness of the method to pathological gait patterns, 2) its agreement with a gold standard, 3) its reliability, and 4) sensitivity to sampling frequency.

1) Subjects: Sixteen persons with PD (14 male; mean (SD) age of 64.4 (11.8) years; disease duration of 12.3 (7.0) years; $\mathrm{H}$ \& Y I: 2, H \& Y II: 8, H \& Y III: 6; and a body height of 173.2 $(8.8) \mathrm{cm}$ ) were involved in this experiment as part of the larger CuPiD study [7]. Coherently with one of the aims of CuPiD, which is to develop a virtual gait trainer for persons with PD that they can use at home in unsupervised conditions, subjects were tested in the ON phase of their anti-Parkinsonian medication.

The study was performed with approval by the Ethical Committee of KU Leuven, in accordance with the Declaration of Helsinki. All subjects provided written informed consent prior to the start of the study.

2) Validity Analysis: For the measurement of spatio-temporal gait parameters, an instrumented 8-m GAITRite (GR) system (CIR Systems Inc., PA) was used as gold standard [29]. GR was here preferred to the treadmill for reasons related to patients safety and comfort. Its sampling rate was set at $120 \mathrm{~Hz}$ and the acquired data were processed using GAITRite Platinum software. Subjects were asked to walk in a straight line, which was guided by the GR mat, while wearing two EXLs1 on their shoes. Once crossed the end of the mat, they stood still for few seconds, then turned back and repeated the test for a total of nine trials. Five of these were performed at a comfortable speed, two at increased speed, and two at decreased speed. The same two IMUs with the same KF settings were used for all participants. IMU data collection was managed via Bluetooth by means of the Android application running on the smartphone (see Section II-F).

For each stride, its duration, length and velocity were obtained both from IMUs and GR. Their level of agreement was quantified by means of Bland-Altman plots [30] and linear regression models.

3) Reliability Analysis: The test-retest reliability of IMUbased parameters was evaluated by computing the Intra-class Correlation Coefficient ICC (type 1,1 [31]) from the five trials at spontaneous speed available for each patient.

4) Sampling Frequency Influence: EXLs1 were all set at a sampling rate of $100 \mathrm{~Hz}$ except on a subset of six patients that had sensors streaming data at $200 \mathrm{~Hz}$. The raw signals recorded on these six patients were then also downsampled to $100 \mathrm{~Hz}$ with a cubic spline interpolation. From these two dataset, it was then possible to infer the influence of sampling frequency on the estimation of spatio-temporal gait parameters.

All data acquired on experiments \#1 and \#2 were processed with the MATLAB-based software version.

\section{RESULTS}

\section{A. Experiment \#1}

The total number of strides acquired on the 12 healthy subjects walking at three different speeds on the treadmill was 1259. 
TABLE I

Summary of TRials AND Gait CyCles AS ObTaINED Form THE 12 HEALTHY SuBJeCtS OF EXPERIMENT \#1 AND THE 14 PD PATIENTS OF EXPERIMENT \#2. Mean \pm 1SD VALUES OF STRIDE DURATION, LENGTH AND Velocity as Measured by the Gold Standard ARE Also Reported

\begin{tabular}{|c|c|c|c|c|c|c|}
\hline & $\mathrm{Ga}$ & $\begin{array}{c}\text { Trials } \\
\text { per } \\
\text { subject }\end{array}$ & $\begin{array}{c}\text { Total } \\
\text { number } \\
\text { of } \\
\text { strides } \\
\end{array}$ & $\begin{array}{l}\text { Mean } \pm 1 \text { SD } \\
\text { stride } \\
\text { duration [s] }\end{array}$ & $\begin{array}{l}\text { Mean } \pm \text { 1SD } \\
\text { stride } \\
\text { length [cm] }\end{array}$ & $\begin{array}{c}\text { Mean } \pm 1 \mathrm{SD} \\
\text { stride } \\
\text { velocity } \\
{[\mathrm{cm} / \mathrm{s}]}\end{array}$ \\
\hline \multirow{4}{*}{$\begin{array}{c}\text { Exp } \\
\# 1\end{array}$} & $1.8 \mathrm{Km} / \mathrm{h}$ & 1 & 379 & 0.2 & 0.6 & $47.9 \pm 2.5$ \\
\hline & $2.7 \mathrm{Km} / \mathrm{h}$ & 1 & 406 & $1.4 \pm 0.1$ & $=7.5$ & $71.6 \pm 3.4$ \\
\hline & $3.5 \mathrm{Km} / \mathrm{h}$ & 1 & 474 & $1.2 \pm 0.1$ & $108.9 \pm 7.8$ & $94.0 \pm 5.2$ \\
\hline & Total & 3 & 1259 & & 6.0 & $72.9 \pm 19.3$ \\
\hline \multirow{4}{*}{$\begin{array}{c}\text { Exp } \\
\# 2\end{array}$} & Comfor & $=$ & 732 & 0.1 & 20.7 & $109.4 \pm 24.7$ \\
\hline & Increased & 2 & 248 & $1.0 \pm 0.1$ & $143.7 \pm 27.9$ & $148.4 \pm 38.0$ \\
\hline & Decreas & 2 & 334 & $1.4 \pm 0.2$ & $111.1 \pm 16.7$ & $79.7 \pm 19.4$ \\
\hline & Total & 9 & 1314 & $1.2 \pm 0.2$ & $124.7 \pm 23.9$ & $109.2 \pm 34.9$ \\
\hline
\end{tabular}

Table I reports the number of gait cycles collected with respect to the three different treadmill speeds, and the mean $\pm 1 \mathrm{SD}$ values of stride duration, length and velocity as obtained from the videocamera. The root mean square (RMS) of the differences between IMU-based and camera-based stride length estimation was: $3.7 \pm 1 \mathrm{~cm}$ at $1.8 \mathrm{Km} / \mathrm{h}, 4.2 \pm 0.9 \mathrm{~cm}$ at $2.7 \mathrm{Km} / \mathrm{h}$ and $5.0 \pm 1 \mathrm{~cm}$ at $3.5 \mathrm{Km} / \mathrm{h}$. The overall RMS of the differences normalized to the mean stride length was equal to $4.0 \%$.

\section{B. Experiment \#2}

One-hundred-twenty-six trials, corresponding to 14 subjects times 9 trials, were correctly collected. Two subjects were excluded from the analysis because of the poor quality of their signals, due to loose fastening of sensors to the patients' shoes. Analyses run independently for each foot sensor. Each trial on the GR mat consisted of 4-9 gait cycles per side, summing up to a total of 1314 gait cycles that could be analyzed. On average, the comfortable speed trial was performed at $4.1 \mathrm{Km} / \mathrm{h}$, the increased speed at $5.6 \mathrm{Km} / \mathrm{h}$ and the decreased speed at $2.9 \mathrm{Km} / \mathrm{h}$.

Table I reports the number of trials and gait cycles collected and the mean $\pm 1 \mathrm{SD}$ values of stride duration, length and velocity as obtained from the GR. The RMS difference between IMU-based and GR stride length estimations was: $3.3 \mathrm{~cm}$ at comfortable walking speed, $4.9 \mathrm{~cm}$ at increased and $3.1 \mathrm{~cm}$ at decreased. The overall RMS of the differences in percentage of the mean stride length was equal to $2.9 \%$.

Fig. 4 shows on a single stride basis the agreement between IMUs and GR in Bland-Altman plots (physical dimensions). Correspondingly, the mean \pm 1SD difference between the two systems in percentage of mean strides duration, length and velocity was: $0.47 \pm 8 \%, 0.14 \pm 5 \%$ and $-0.33 \pm 10 \%$, respectively. Besides, Fig. 5 shows the linear regression models between the values obtained from the two systems.

The mean of stride duration, stride length, and gait speed for each of the five walking trials at comfortable speed was used to determine the test-retest reliability by means of ICC (type 1,1) with $95 \%$ confidence intervals. Results in Table II show all ICC values above 0.9 that, according to the benchmarks suggested by [29], qualify the reliability of all spatio-temporal measures as excellent.

The mean $\pm 1 \mathrm{SD} \%$ difference between the values of mean stride duration estimated at the two sampling frequencies was
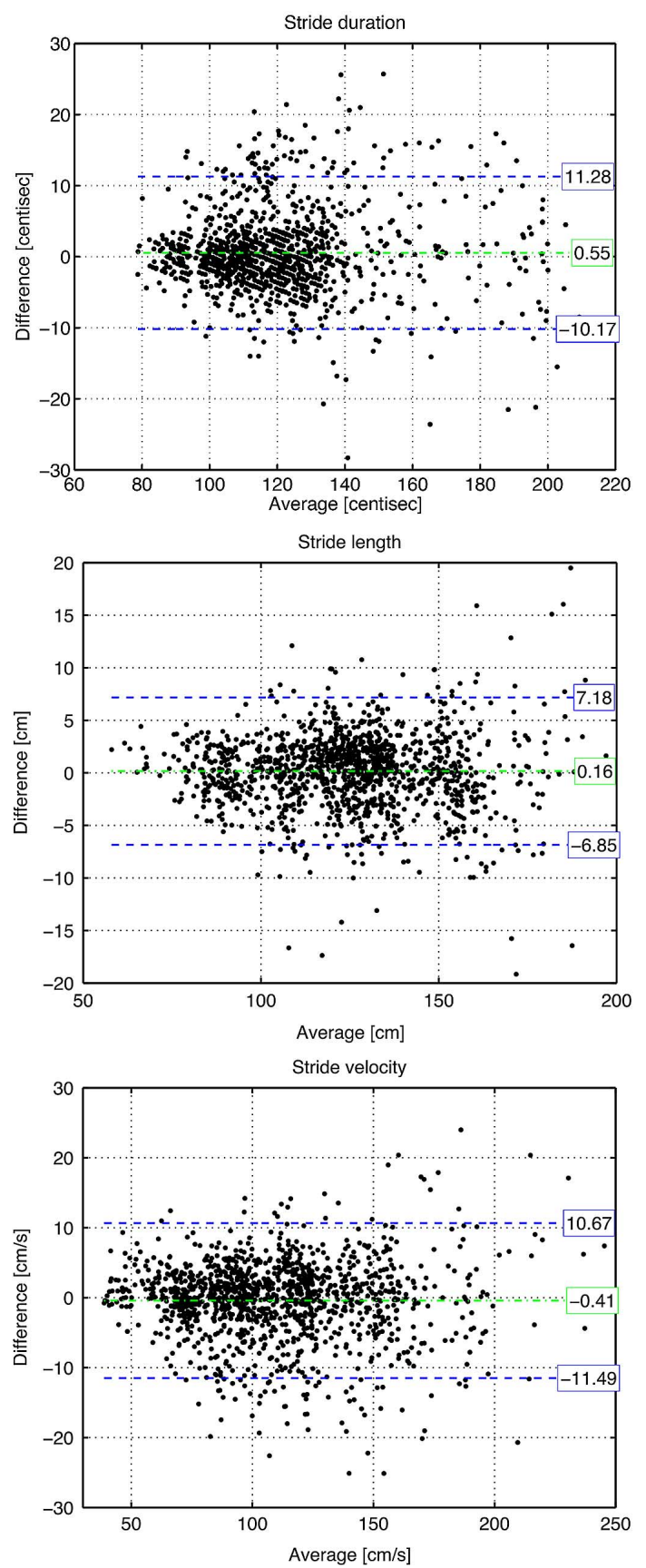

Fig. 4. Bland-Altman comparison plots. The x-axis shows the arithmetic mean of results obtained with the system proposed in this paper against the gold standard; the y-axis shows their differences in physical dimension with respect to the stride duration (first plot), the stride length (second plot) and the stride velocity (third plot). Each plot reports 1314 dots in correspondence to each gait cycle collected on the 14 PD subjects enrolled. The dash-dotted line in the middle is the arithmetic mean of the differences, the dashed lines above and below denotes $1 \mathrm{SD}$.

$0.43 \pm 8.3 \%$ at $100 \mathrm{~Hz}$ and $0.43 \pm 8.3 \%$ at $200 \mathrm{~Hz}$. Correspondingly, mean $\pm 1 \mathrm{SD} \%$ difference in mean stride length was $0.58 \pm 6.1 \%$ at $100 \mathrm{~Hz}$ and $0.86 \pm 3.7 \%$ at $200 \mathrm{~Hz}$.

\section{Porting on a Smartphone}

Fig. 6 shows the resulting layout of the Android app developed to provide the spatio-temporal gait parameters in real-time. 

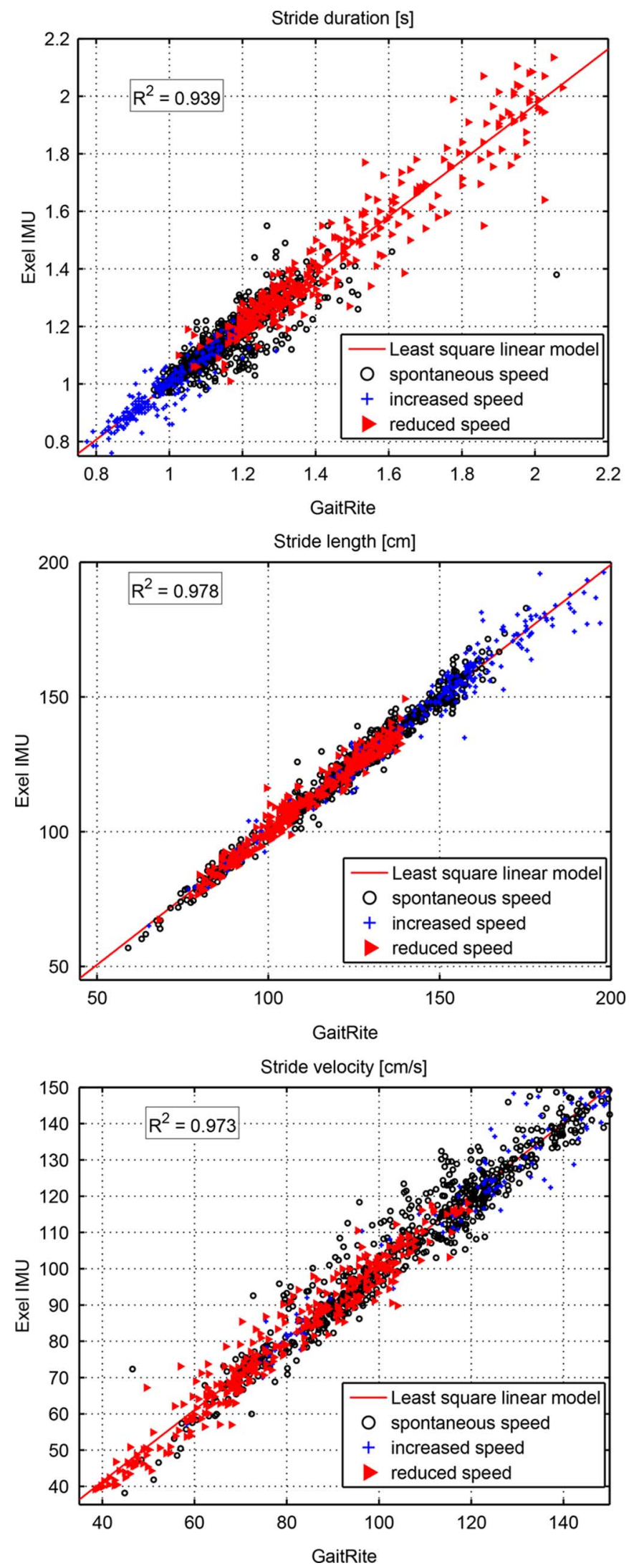

Fig. 5. Regression lines on stride duration (first plot), stride length (second plot), and stride velocity (third plot) of the system proposed in this paper (y-axis) against the gold standard (x-axis) on the 1314 gait cycles collected on the $14 \mathrm{PD}$ subjects enrolled. The circle, plus sign and right-pointing triangle markers represent gait cycles collected, respectively, at spontaneous, increased and reduced walking speed.
TABLE II

TEST-Retest Reliability of Stride Duration, Length and Velocity From the 5 WALKIng Trials at COMFortable SPEED

\begin{tabular}{|c|c|c|c|}
\hline \hline & Stride Duration & Stride Length & Stride Velocity \\
\hline ICC $(1,1)$ & 0.909 & 0.972 & 0.974 \\
CI of ICC & {$[0.820-0.965]$} & {$[0.942-0.990]$} & {$[0.945-0.990]$} \\
\hline \hline
\end{tabular}

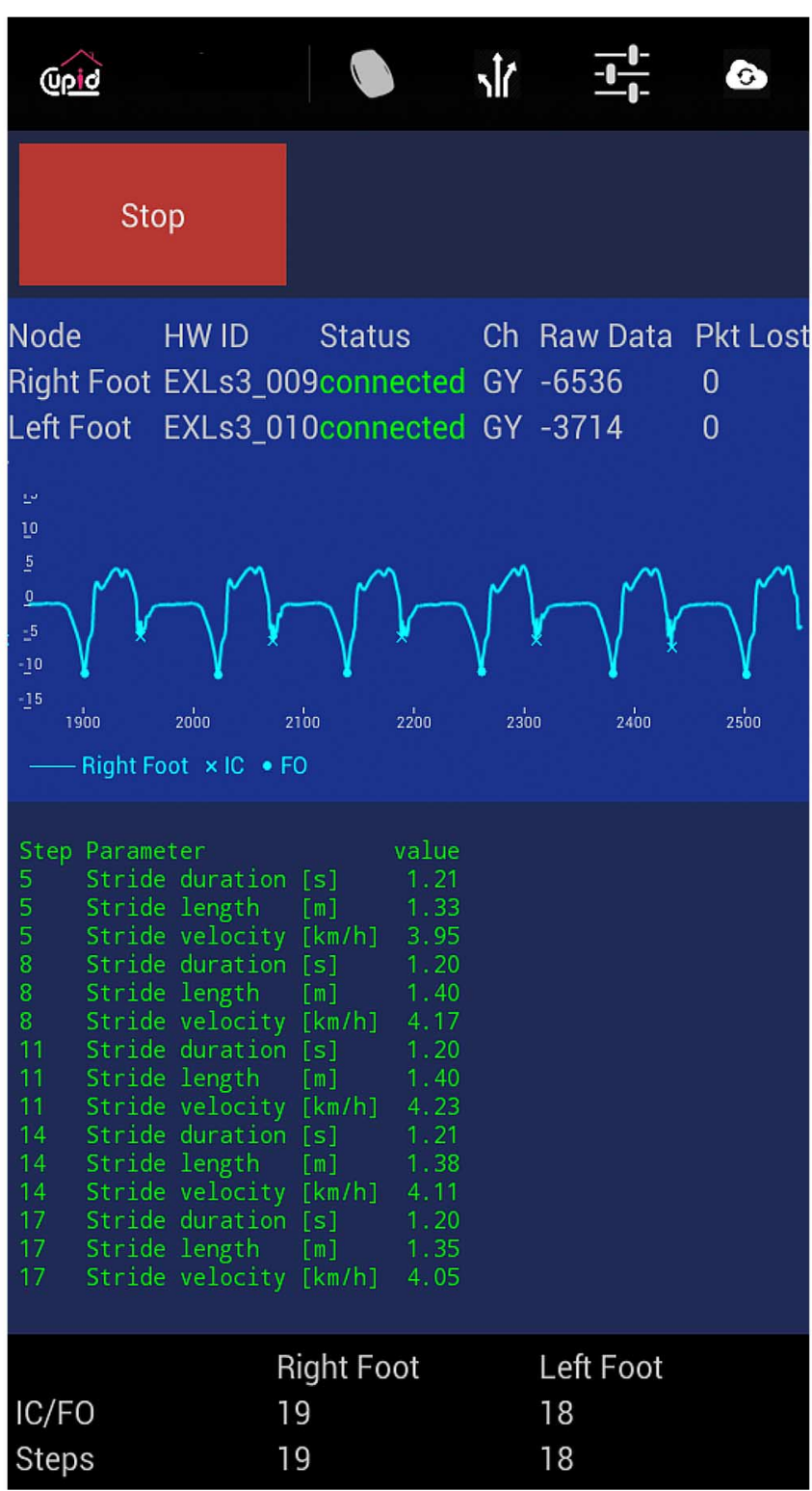

Fig. 6. Screenshot of the Android app developed to provide the spatio-temporal gait parameters in real time.

In particular, the app 1) collects data streamed from the shoeworn IMUs via Bluetooth, 2) logs data retrieved on the smartphone internal memory, 3) runs the algorithms for gait analysis, 4) estimate spatio-temporal gait parameters, 5) plots the angular rate of a single IMU per time with superimposed the IC and FO events, and 6) displays in real-time the values of gait parameters.

The porting of the ZUPT-PDR algorithms in Java did not significantly affect accuracy and precision in foot position reckoning. Fig. 7 shows in a probability plot for normal distribu- 


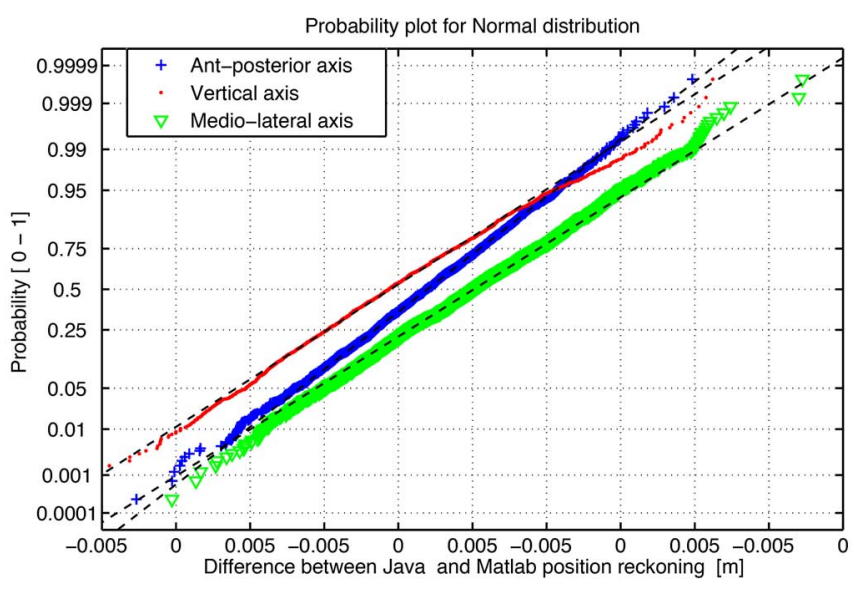

Fig. 7. Probability plot for normal distribution of the differences on position reckoning from MATLAB and Java based PDR algorithms on a total of 2080 frames collected during a trial acquisition of 100 strides. For each frame, the plus sign, the triangle and the dot markers denote the difference relative to, respectively, the antero-posterior, the medio-lateral and the vertical directions.

TABLE III

CPU OCCUPATION ON A SAMSUNG S3Mini DURING THE APP RUNNING

\begin{tabular}{|c|c|c|}
\hline \hline Process & CPU occupation $\%$ & Application relative $\%$ \\
\hline App & $6.4 \%$ & $100 \%$ \\
\hline Text data logging & $3.78 \%$ & $59 \%$ \\
\hline Raw data logging & $0.77 \%$ & $12 \%$ \\
\hline $\begin{array}{c}\text { PDR and gait } \\
\text { parameters estimation }\end{array}$ & $0.70 \%$ & $11 \%$ \\
\hline Bluetooth mgmt. & $0.51 \%$ & $8 \%$ \\
\hline UI mgmt. & $0.19 \%$ & $3 \%$ \\
\hline $\begin{array}{c}\text { Other (message } \\
\text { queues, scheduling) }\end{array}$ & $0.45 \%$ & $7 \%$ \\
\hline \hline
\end{tabular}

tion, the differences on position reckoning between MATLAB and Java versions of the software on a walking trial of 100 steps.

The mobile application was benchmarked to verify CPU occupation on the smartphone. In particular, the app achieved realtime performance and run with no delay; results are shown in Table III.

Memory benchmarks for the application shows occupancy almost constant during the execution; heap memory occupied is $36 \mathrm{Mb}$. To compare Java with MATLAB software version, a stress test was performed on a Samsung S3Mini and a PC with i3 CPU. The phone was capable to process up to 6000 , whereas MATLAB 4000 samples per second. Still, it should be taken into account that the two architectures are very different one another and this comparison is mostly qualitative.

\section{DISCUSSION}

\section{A. Accuracy of the Algorithms Proposed for Spatio-Temporal Gait Parameters Estimation}

Aim of the study was to validate a wearable real-time implementation of a KF ZUPT-aided gait analysis system based on shoe-worn inertial sensors.

The system was first prototyped in MATLAB and then ported as a library to an Android smartphone. The system allows for automatic estimation of gait parameters and their real-time display. More so, it is independent on sensor positioning at the shoe, and allows for maintaining the accuracy across different patients with different gait patterns and walking speeds.

The analysis accomplished in experiment \#1 with healthy subjects walking on a treadmill revealed a good agreement between IMU-based and camera-based step length measurements. This confirms the fact that the values of the internal parameters of the algorithms for IC and FO detection, ZUPT determination and of the KF were optimally tuned. Besides, the three different speeds set on the treadmill did not significantly influence the accuracy on step length estimation.

The focus of this study was however on the design of a system, which prospectively can be used on persons affected by gait disturbances, showing altered and irregular gait patterns. A sample group of 16 persons with Parkinson's disease was then recruited. Figs. 4 and 5 show the system's performances of the detector described in Section II.C and the ZUPT-PDR algorithm outlined in Section II.D and II.E. Both algorithms provided a good agreement with the reference system (GR), even with Parkinsonian gait patterns. Particularly, for stride length and velocity, our results showed better accuracy and precision than previously reported systems based on biomechanical models. For example, [26], [32] reported a precision for stride time and length of respectively $21.1 \%$ and $8.3 \%$; whilst [8] reported an accuracy on step length of $13.0 \%$. Compared to other strap-down integration methods, such as [19], [33], the results we obtained show similar accuracy and precision. However, unlike other methods, the KF allows to avoid assumptions on the type of walked surface (e.g., flat [18]), and, thanks to the recursive nature of the algorithm, to have the localization with a delay of just a single frame.

Increasing the sampling rate to $>100 \mathrm{~Hz}$ did not affect IC and FO detection accuracy, whilst it significantly improved the precision of step length reconstruction dropping from a SD of $6.1 \%$ at $100 \mathrm{~Hz}$ to $3.7 \%$ at $200 \mathrm{~Hz}$. The walking speeds expressed by patients in experiment $\# 2$ were unexpectedly higher than the ones set beforehand on the treadmill for volunteers in experiment \#1. By looking at the raw data of the EXLs1 accelerometer in the increased speed trials of patients in experiment $\# 2$, walking speeds above $150 \mathrm{~cm} / \mathrm{s}$ have probably determined the saturation of the accelerometer and as a consequence a loss of accuracy in stride length and velocity estimation. For this reason the full scales of $\pm 1000^{\circ} / \mathrm{s}$ (for the gyroscope) and of $\pm 8 \mathrm{~g}$ (for the accelerometer) available on the EXLs3 IMUs appeared the most suitable to best sample gait pattern and speed ranges as those expressed in this study.

The porting of the ZUPT-PDR algorithm in Java did not affect accuracy and precision in spatial parameters estimation (Fig. 7). The slight difference observed between the two algorithms can be explained with different variable representation and approximation when executing the computation. The systems on which the two algorithms are executed have indeed different microprocessor architecture, and make use of different mathematical libraries. Some skewness can be observed and is more evident on the vertical axis; this is due to the fact that the motion on the vertical axis is less intense with respect to the forward axis, and the vertical axis is more subject (with respect 
to other axes) to approximation in the algorithm that compute the acceleration of gravity in the location where measurement is performed. In conclusion, the off-the-shelf smartphone used in this study demonstrated the ability of the app to handle real-time spatio-temporal gait parameters estimation and visualization.

\section{B. Fields of Application of the System and Algorithms Developed}

Considering the unobtrusive sensor setup, the cost effectiveness of its components, the real-time operation, and its easy deployment to new environments, this system appears well suited for supporting persons with gait disturbances in their daily life. The only requirement of the ZUPT-PDR algorithm is a proper detection of IC and FO events. This application can thus be used as long as patients are not severely impaired (when their shuffling gait could mask IC and FO events). A proficient use of the system is therefore likely to apply also on patients with other gait disturbances, e.g., originating from Stroke or other movement disorders.

Recent studies, have shown how feedback and intensive exercises may enhance and speed up the processes of acquisition, automation and retention of motor skills such as walking [5], [6], [34]. Also, aerobic walking in a community setting is safe, well tolerated, and improves aerobic fitness, motor function, endurance to fatigue, mood, executive control, and quality of life [35].

Exploiting the features of full portability, ease-of-use and accuracy in providing real-time feedback on the user's gait, systems like the one proposed in this study have the potential of a breakthrough in the rehabilitation field by enabling standalone provision of motor exercises with continuous supervision and monitoring. For example, such a system could continuously guide and supervise the gait performance of PD patients during outdoor transfers, e.g., in terms of step length, asymmetry or speed, acting as an intelligent tutoring systems [25]. Ultimately, similar solutions will allow for high-intensity taskspecific training on single or multiple spatio-temporal gait parameters, implementing long-term rehabilitation programs [36], [37].

\section{CONCLUSION}

New methods have been proposed and described for estimating spatio-temporal gait parameters using shoe-worn inertial sensors and a smartphone. The algorithms for initial-contact and foot-off detection and for stride length and velocity estimation were tuned on healthy volunteers and validated on persons with Parkinson's disease against a reference electronic walkway system. The results prove that these algorithms are able to provide accurate spatio-temporal gait parameters estimation complying with different gait patterns and velocities.

This study provides new insight into the exploitation of PDR methods and KF towards enabling stand-alone provision of gait exercises with continuous supervision and monitoring by means of fully wearable systems.

\section{ACKNOWLEDGMENT}

The authors would like to thank the academic project OpenShoe that constituted a useful basis for methods implementation, as well as ETH students D. Clausen and S. Mach for their contribution in data collection.

\section{REFERENCES}

[1] P. Langhorne, F. Coupar, and A. Pollock, "Motor recovery after stroke: A systematic review," Lancet Neurol., vol. 8, no. 8, pp. 741-754, Aug. 2009.

[2] J. Jankovic, "Parkinson's disease: Clinical features and diagnosis," $J$ Neurol. Neurosurg. Psychiatry, vol. 79, no. 4, pp. 368-376, Apr. 2008.

[3] J. M. Hausdorff, “Gait dynamics in Parkinson's disease: Common and distinct behavior among stride length, gait variability, and fractal-like scaling," Chaos, vol. 19, no. 2, p. 026113, Jun. 2009.

[4] A.-L. Hsu, P.-F. Tang, and M.-H. Jan, "Analysis of impairments influencing gait velocity and asymmetry of hemiplegic patients after mild to moderate stroke," Arch. Phys. Med. Rehabil., vol. 84, no. 8, pp. 1185-1193, Aug. 2003.

[5] A. Nieuwboer, L. Rochester, L. Müncks, and S. P. Swinnen, "Motor learning in Parkinson's disease: Limitations and potential for rehabilitation," Parkinsonism Relat. Disord., vol. 15, pp. S53-58, Dec. 2009.

[6] P. B. Shull, W. Jirattigalachote, M. A. Hunt, M. R. Cutkosky, and S. L. Delp, "Quantified self and human movement: A review on the clinical impact of wearable sensing and feedback for gait analysis and intervention," Gait Posture, vol. 40, no. 1, pp. 11-19, May 2014.

[7] CuPiD, "Closed-loop system for personalized and at-home rehabilitation of people with Parkinson's disease," 2011-2014, FP7-ICT-288516.

[8] S. Hundza et al., "Accurate and reliable gait cycle detection in Parkinson's disease,” IEEE Trans. Neural Syst. Rehabil. Eng., vol. 22, no. 1, pp. 127-137, Oct. 2013.

[9] J. K. Lee and E. J. Park, "Quasi real-time gait event detection using shank-attached gyroscopes," Med. Biol. Eng. Comput., vol. 49, no. 6, pp. 707-712, Jun. 2011

[10] D. T.-P. Fong and Y.-Y. Chan, "The use of wearable inertial motion sensors in human lower limb biomechanics studies: A systematic review," Sensors (Basel), vol. 10, no. 12, pp. 11556-11565, Dec. 2010.

[11] J. Rueterbories, E. G. Spaich, B. Larsen, and O. K. Andersen, "Methods for gait event detection and analysis in ambulatory systems," Med. Eng. Phys., vol. 32, no. 6, pp. 545-552, Jul. 2010.

[12] S. Yang and Q. Li, "Inertial sensor-based methods in walking speed estimation: A systematic review," Sensors (Basel), vol. 12, no. 5, pp. 6102-6116, Jan. 2012.

[13] K. Aminian, B. Najafi, C. Büla, P.-F. Leyvraz, and P. Robert, "Spatiotemporal parameters of gait measured by an ambulatory system using miniature gyroscopes," J. Biomech., vol. 35, no. 5, pp. 689-699, May 2002.

[14] A. M. A. Sabatini, "Quaternion-based strap-down integration method for applications of inertial sensing to gait analysis," Med. Biol. Eng. Comput., vol. 43, no. 1, pp. 94-101, Feb. 2005.

[15] E. Foxlin, "Pedestrian tracking with shoe-mounted inertial sensors," IEEE Comput. Graph. Appl., vol. 25, no. 6, pp. 38-46, Nov. 2005.

[16] J. Perry, Gait Analysis: Normal and Pathological Function. Thorofare, NJ: Slack, 1992.

[17] J.-O. Nilsson, I. Skog, P. Handel, and K. V. S. Hari, "Foot-mounted INS for everybody-An open-source embedded implementation," Proc. PLANS, pp. 140-145, 2012.

[18] B. Mariani, M. C. Jiménez, F. J. G. Vingerhoets, and K. Aminian, “Onshoe wearable sensors for gait and turning assessment of patients with Parkinson's disease," IEEE Trans. Biomed. Eng., vol. 60, no. 1, pp. 155-158, Jan. 2013.

[19] A. Rampp et al., "Inertial sensor based stride parameter calculation from gait sequences in geriatric patients," IEEE Trans. Biomed. Eng., vol. 62, no. 4, pp. 1089-1097, Apr. 2015.

[20] C. Fischer, P. Talkad Sukumar, and M. Hazas, "Tutorial: Implementing a Pedestrian tracker using inertial sensors," IEEE Pervasive Comput., vol. 12, no. 2, pp. 17-27, Apr. 2013.

[21] J.-O. Nilsson, I. Skog, and P. Handel, "A note on the limitations of ZUPTs and the implications on sensor error modeling," in Proc. IPIN, 2012, pp. 13-15.

[22] I. Skog, J.-O. Nilsson, and H. Peter, "Evaluation of zero-velocity detectors for foot-mounted inertial navigation systems," in Proc. IPIN, 2010, pp. 15-17.

[23] C. Franco et al., "iBalance-ABF: A smartphone-based audio-biofeedback balance system," IEEE Trans. Biomed. Eng., vol. 60, no. 1, pp. 211-215, Jan. 2013. 
[24] M. A. Hobert, W. Maetzler, K. Aminian, and L. Chiari, "Technical and clinical view on ambulatory assessment in Parkinson's disease," Acta Neurol. Scand., vol. 130, no. 3, pp. 139-147, Sept. 2014.

[25] F. Casamassima et al., "A wearable system for gait training in subjects with Parkinson's disease," Sensors (Basel), vol. 14, no. 4, pp. 6229-6246, Mar. 2014.

[26] A. Salarian et al., "Gait assessment in Parkinson's disease: Toward an ambulatory system for long-term monitoring," IEEE Trans. Biomed. Eng., vol. 51, no. 8, pp. 1434-1443, Aug. 2004.

[27] L. R. Gauthier, M. E. Jansen, and J. R. Meyer, "Single video camera method for using scene metrics to measure constrained 3D displacements," in Proc. SPIE, 2014.

[28] A. Matsas, N. Taylor, and H. McBurney, "Knee joint kinematics from familiarised treadmill walking can be generalised to overground walking in young unimpaired subjects," Gait Posture, vol. 11, no. 1, pp. 46-53, Feb. 2000.

[29] H. B. Menz, M. D. Latt, A. Tiedemann, M. Mun San Kwan, and S. R. Lord, "Reliability of the GAITRite walkway system for the quantification of temporo-spatial parameters of gait in young and older people," Gait Posture, vol. 20, no. 1, pp. 20-25, Aug. 2004.

[30] J. M. Bland and D. G. Altman, "Statistical methods for assessing agreement between two methods of clinical measurement," Lancet, vol. 1, no. 8476, pp. 307-310, Feb. 1986.

[31] K. O. McGraw and S. P. Wong, "Forming inferences about some intraclass correlation coefficients," Psychol. Methods, vol. 50, no. 1, pp. 30-46, 1996.
[32] K. Aminian et al., "Evaluation of an ambulatory system for gait analysis in hip osteoarthritis and after total hip replacement," Gait Posture, vol. 20, no. 1, pp. 102-107, Aug. 2004.

[33] B. Mariani et al., "3D gait assessment in young and elderly subjects using foot-worn inertial sensors," J. Biomech., vol. 43, no. 15, pp. 2999-3006, Nov. 2010.

[34] A. Nieuwboer et al., "Cueing training in the home improves gait-related mobility in Parkinson's disease: The RESCUE trial," J. Neurol. Neurosurg. Psychiatry, vol. 78, no. 2, pp. 134-140, Feb. 2007.

[35] E. Y. Uc et al., "Phase I/II randomized trial of aerobic exercise in Parkinson disease in a community setting," Neurology, vol. 83, no. 5, pp. 413-425, Jul. 2014.

[36] K. Winfree, I. Pretzer-Aboff, and D. Hilgart, "Effect of step-synchronized vibration on patients with Parkinson's disease: Case studies on subjects with freezing of gait or an implanted deep brain stimulator," IEEE Trans. Neural Syst. Rehabil. Eng., vol. 21, no. 5, pp. 806-811, Sep. 2013

[37] F. B. Horak, M. Dozza, R. Peterka, L. Chiari, and C. Wall, "Vibrotactile biofeedback improves tandem gait in patients with unilateral vestibular loss," Ann. NY Acad. Sci., vol. 1164, pp. 279-281, May 2009.

Author's photographs and biographies not available at the time of publication. 